\title{
Models of magnetized neutron star atmospheres: thin atmospheres and partially ionized hydrogen atmospheres with vacuum polarization
}

\author{
V. Suleimanov ${ }^{1,2}$, A. Y. Potekhin ${ }^{3,4}$, and K. Werner ${ }^{1}$ \\ ${ }^{1}$ Institut für Astronomie und Astrophysik, Kepler Center for Astro and Particle Physics, Universität Tübingen, Sand 1, \\ 72076 Tübingen, Germany \\ e-mail: suleimanov@astro.uni-tuebingen.de \\ 2 Kazan State University, Kremlevskaja str., 18, Kazan 420008, Russia \\ 3 Ioffe Physical-Technical Institute, Polytekhnicheskaya str., 26, St. Petersburg 194021, Russia \\ 4 Isaac Newton Institute of Chile, St. Petersburg Branch, Russia
}

Received 20 March 2009 / Accepted 14 April 2009

\begin{abstract}
Context. Observed X-ray spectra of some isolated magnetized neutron stars display absorption features, sometimes interpreted as ion cyclotron lines. Modeling the observed spectra is necessary to check this hypothesis and to evaluate neutron star parameters.

Aims. We develop a computer code for modeling magnetized neutron star atmospheres in a wide range of magnetic fields $\left(10^{12}-10^{15} \mathrm{G}\right)$ and effective temperatures $\left(3 \times 10^{5}-10^{7} \mathrm{~K}\right)$. Using this code, we study the possibilities to explain the soft X-ray spectra of isolated neutron stars by different atmosphere models.

Methods. The atmosphere is assumed to consist either of fully ionized electron-ion plasmas or of partially ionized hydrogen. Vacuum resonance and partial mode conversion are taken into account. Any inclination of the magnetic field relative to the stellar surface is allowed. We use modern opacities of fully or partially ionized plasmas in strong magnetic fields and solve the coupled radiative transfer equations for the normal electromagnetic modes in the plasma.

Results. Spectra of outgoing radiation are calculated for various atmosphere models: fully ionized semi-infinite atmosphere, thin atmosphere, partially ionized hydrogen atmosphere, or novel "sandwich" atmosphere (thin atmosphere with a hydrogen layer above a helium layer). Possibilities of applications of these results are discussed. In particular, the outgoing spectrum using the "sandwich" model is constructed. Thin partially ionized hydrogen atmospheres with vacuum polarization are shown to be able to improve the fit to the observed spectrum of the nearby isolated neutron star RBS 1223 (RX J1308.8+2127).
\end{abstract}

Key words. stars: individual: RX J1308.8+2127- radiative transfer - methods: numerical - stars: neutron - stars: atmospheres - X-rays: stars

\section{Introduction}

In the last two decades, several new classes of neutron stars (NSs) have been discovered by X-ray observatories. They include X-ray dim isolated NSs (XDINSs), or Magnificent Seven (see review by Haberl 2007), central compact objects (CCOs) in supernova remnants (Pavlov et al. 2002, 2004), anomalous X-ray pulsars and soft-gamma repeaters (AXPs and SGRs; see reviews by Kaspi 2007; Mereghetti et al. 2007; Mereghetti 2008). The NSs in the last two classes have superstrong magnetic fields $\left(B \gtrsim 10^{14} \mathrm{G}\right)$ and are commonly named magnetars. The XDINSs can have $B \sim$ a few $\times 10^{13} \mathrm{G}$, as evaluated from period changes and from absorption features in the observed spectra, if they are interpreted as ion cyclotron lines (see reviews by Haberl 2007; van Kerkwijk \& Kaplan 2007).

These NSs are relatively young with ages $\leq 10^{6} \mathrm{yr}$ and sufficiently hot $\left(T_{\text {eff }} \sim 10^{6}-10^{7} \mathrm{~K}\right)$ to be observed as soft X-ray sources. The thermal spectra of these objects can be described by blackbody spectra with (color) temperatures from 40 to $700 \mathrm{eV}$ (see, for example, Mereghetti et al. 2002). Some of the XDINSs and CCOs in supernova remnants have one or more absorption features in their X-ray spectrum at the energies $0.2-0.8 \mathrm{keV}$ (Haberl 2007). The central energies of these features appear to be harmonically spaced (Sanwal et al. 2002; Schwope et al. 2007; van Kerkwijk \& Kaplan 2007; Haberl 2007). The optical counterparts of some XDINSs are also known (see review by Mignani et al. 2007), and their optical/ultraviolet fluxes are a few times larger than the blackbody extrapolation of the X-ray spectra (Burwitz et al. 2001, 2003; Kaplan et al. 2003; Motch et al. 2003).

The XDINs are nearby objects, and parallaxes of some of them can be measured (Kaplan et al. 2002a). Therefore, they give a good possibility to measure the NS radii, yielding useful information on the equation of state (EOS) for the NS inner core (Trümper et al. 2004; Lattimer \& Prakash 2007), which is one of the most important problems in the NS physics. For example, the EOS is necessary for computations of templates of gravitational wave signals which arise during neutron stars merging (e.g. Baiotti et al. 2008).

For a sufficiently accurate evaluation of NS radii, a good model of the NS surface radiation for the observed X-ray spectra fitting is necessary. The isolated NS surface layers can either be condensed or have a plasma envelope (Lai \& Salpeter 1997; Lai 2001). In the latter case, the outer envelope layer forms an NS atmosphere. The structure and emergent spectrum of this atmosphere can be computed by using stellar model 
atmosphere methods (e.g. Mihalas 1978). Such modeling has been performed by many scientific groups for NS model atmospheres without magnetic field (Romani 1987; Zavlin et al. 1996; Rajagopal \& Romani 1996; Werner \& Deetjen 2000; Gänsicke et al. 2002; Pons et al. 2002) and by several groups for models with strong $\left(B \gtrsim 10^{12} \mathrm{G}\right)$ magnetic fields (Shibanov et al. 1992; Rajagopal et al. 1997; Özel 2001; Ho \& Lai 2001, 2003, 2004; van Adelsberg \& Lai 2006). These model spectra were used to fit the observed isolated NS X-ray spectra (see review by Zavlin 2009).

Modeling of magnetized NS star atmospheres is based on the theory of electromagnetic wave propagation in a magnetized plasma in two normal modes, extraordinary (X) and ordinary (O) ones (Ginzburg 1970; Mésźaros 1992), and on the methods of opacity calculations for these two modes (Ventura 1979; Kaminker et al. 1982, 1983). Methods of fully ionized model atmospheres modeling are well developed (see, e.g., Zavlin 2009 for references). Partially ionized hydrogen atmospheres have been modeled (Potekhin et al. 2004; Ho \& Lai 2004; Ho et al. 2008), using the opacity and EOS calculations by Potekhin \& Chabrier (2003, 2004), which accurately take into account the motional Stark effect and plasma nonideality effects in quantizing magnetic fields. Mid- $Z$ element atmospheres for strongly magnetized NSs have been modeled by Mori \& Ho (2007), who treat the motional Stark effect using a perturbation theory (Pavlov \& Mészáros 1993) valid at relatively low $T$.

For magnetar atmospheres, polarization of the vacuum can be significant, which was studied by Pavlov \& Gnedin (1984) and recently by Lai \& Ho $(2002,2003)$. Model atmospheres with partial mode conversion due to the vacuum polarization have been computed by Ho \& Lai (2003) and van Adelsberg \& Lai (2006).

If the temperature is sufficiently low or the magnetic field is sufficiently strong, the thick atmosphere can be replaced by a condensed surface (Lai \& Salpeter 1997; Lai 2001; Medin \& Lai 2007) as a result of the plasma phase transition (cf. Potekhin et al. 1999; Potekhin \& Chabrier 2004). Emission and absorption properties of such surfaces in strong magnetic fields have been studied by Turolla et al. (2004), van Adelsberg et al. (2005), and Pérez-Azorín et al. (2005).

In recent years, evidence has appeared that some of the XDINSs may have a "thin" atmosphere above the condensed surface. Such atmosphere could be optically thick to low-energy photons and optically thin to high-energy photons. Motch et al. (2003) fitted the spectrum of RX J0720.4-3125 using a nonmagnetic thin hydrogen atmosphere model, and Ho et al. (2007) fitted the spectrum of RX J1856.4-3754 using a magnetic, partially ionized, thin hydrogen atmosphere model.

In this paper we present a new computer code for model atmospheres of magnetized NSs and some new results, which were obtained by using this code. The code is applicable to modeling of fully ionized NS atmospheres and partially ionized hydrogen atmospheres accounting for the partial mode conversion due to the vacuum polarization. The magnetic field can be normal or inclined to NS surface. We use this code to study various thin atmospheres above a condensed NS surface. In particular, we consider a novel "sandwich" atmosphere model, where a thin atmosphere is composed of a hydrogen layer above a helium layer. We also discuss the possibility to apply our thin partially ionized hydrogen model atmospheres with vacuum polarization to fit the spectrum of RBS 1223. Our numerical method is outlined in Sect. 2. Results are presented and discussed in Sect. 3. Conclusions are summarized in Sect. 4.

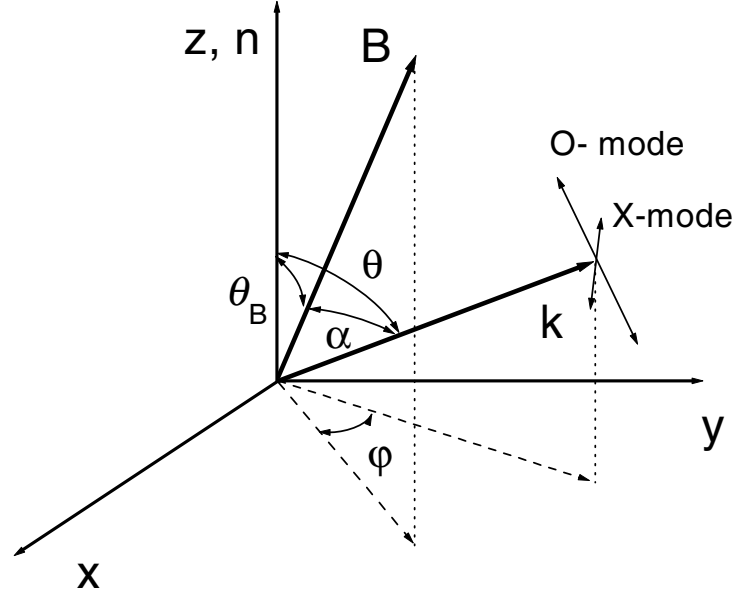

Fig. 1. Geometry of the radiation transfer.

\section{Method of atmosphere structure calculations}

We compute model atmospheres of hot, magnetized NSs subject to the constraints of hydrostatic and radiative equilibrium assuming planar geometry and homogeneous magnetic field. There are two versions of the code. In the first one, we consider the magnetic field $\boldsymbol{B}$ perpendicular to the surface. In this case the angle $\alpha$ between $\boldsymbol{B}$ and a radiation wave vector $\boldsymbol{k}$ is equal to the angle $\theta$ between $\boldsymbol{k}$ and the normal $\boldsymbol{n}$ to the surface (see Fig. 1). It is the simplest case, because opacities depend on $\alpha$, and the geometry of radiation propagation depends on $\theta$. In the second version, the angle $\theta_{B}$ between $\boldsymbol{B}$ and $\boldsymbol{n}$ is arbitrary, and calculations are more expensive. In this case the opacities depend not only on the polar angle $\theta$, but also on the azimuthal angle $\varphi$ between the projections of $\boldsymbol{B}$ and $\boldsymbol{n}$ onto the stellar surface, therefore it is necessary to solve radiation transfer equations for a significantly larger number of directions.

The model atmosphere structure for a NS with effective temperature $T_{\text {eff }}$, surface gravity $g$, magnetic field $B$, and given chemical composition is described by the following set of equations:

1. The hydrostatic equilibrium equation

$\frac{\mathrm{d} P_{\mathrm{g}}}{\mathrm{d} m}=g-g_{\mathrm{rad}}$

where

$$
\begin{aligned}
g & =\frac{G M_{\mathrm{NS}}}{R_{\mathrm{NS}}^{2} \sqrt{1-R_{\mathrm{S}} / R_{\mathrm{NS}}}} \\
g_{\mathrm{rad}} & =\frac{1}{c} \sum_{i=1}^{2} \int_{0}^{\infty} \mathrm{d} v \int_{0}^{2 \pi} \mathrm{d} \varphi \int_{-1}^{+1}\left(k_{v}^{i}+\sigma_{v}^{i}\right) \mu I_{v}^{i}(\mu, \varphi) \mathrm{d} \mu
\end{aligned}
$$

allows for the radiation pressure, and $R_{\mathrm{S}}=2 G M_{\mathrm{NS}} / c^{2}$ is the Schwarzschild radius of the NS. Here $\mu=\cos \theta, I_{v}^{i}(\mu, \varphi)$ is the specific intensity in mode $i, P_{\mathrm{g}}$ is the gas pressure, and the column density $m$ is determined as

$\mathrm{d} m=-\rho \mathrm{d} z$.

The variable $\rho$ denotes the gas density and $z$ is the vertical distance. Radiation pressure is unimportant in the models presented below, but it can be significant at higher effective temperatures. 


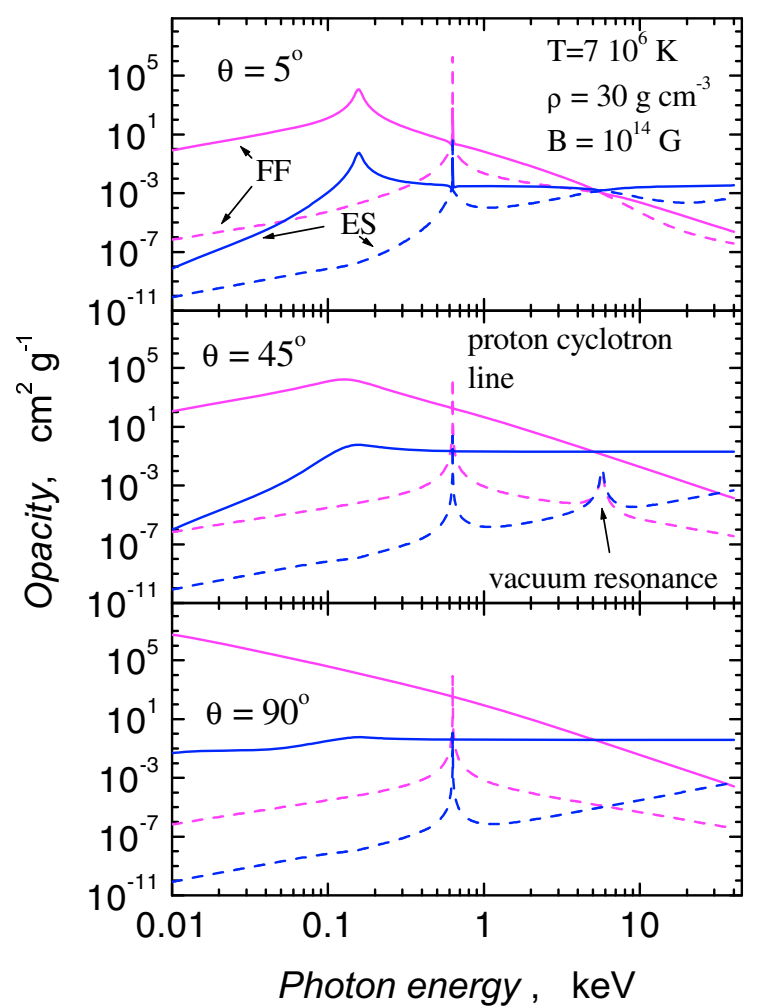

Fig. 2. Dependence of the free-free and electron scattering opacities in two modes on the photon energy at different angles in a fully ionized hydrogen plasma. The proton cyclotron and vacuum resonances are also shown. The plasma temperature is $7 \times 10^{6} \mathrm{~K}$, the plasma density is $30 \mathrm{~g} \mathrm{~cm}^{-3}$, the magnetic field strength is $10^{14} \mathrm{G}$.

2. The radiation transfer equations for the two modes

$\mu \frac{\mathrm{d} I_{v}^{i}}{\mathrm{~d} \tau_{v}^{i}}=I_{v}^{i}-S_{v}^{i}$

where

$$
\begin{aligned}
& S_{v}^{i}=\frac{k_{v}^{i}}{k_{v}^{i}+\sigma_{v}^{i}} \frac{B_{v}}{2}+ \\
& \frac{1}{2 \pi} \frac{1}{k_{v}^{i}+\sigma_{v}^{i}} \sum_{j=1}^{2} \int_{0}^{2 \pi} \mathrm{d} \varphi^{\prime} \int_{-1}^{+1} \sigma_{v}^{i j}\left(\mu, \varphi ; \mu^{\prime}, \varphi^{\prime}\right) I_{v}^{j}\left(\mu^{\prime}, \varphi^{\prime}\right) \mathrm{d} \mu^{\prime}
\end{aligned}
$$

is the source function, $B_{v}$ is the blackbody (Planck) intensity, and the optical depth $\tau_{v}^{i}$ is defined as

$\mathrm{d} \tau_{v}^{i}=\left(k_{v}^{i}+\sigma_{v}^{i}\right) \mathrm{d} m$

Here, the true absorption and electron scattering opacities $k_{v}^{i}$ and $\sigma_{v}^{i}$ depend on $\mu$ and $\varphi$. Specific intensity in given direction and mode can be scattered in some other direction and in both modes

$$
\sigma_{v}^{i}(\mu, \varphi)=\frac{1}{2 \pi} \sum_{j=1}^{2} \int_{0}^{2 \pi} \mathrm{d} \varphi^{\prime} \int_{-1}^{+1} \sigma_{v}^{i j}\left(\mu, \varphi ; \mu^{\prime}, \varphi^{\prime}\right) \mathrm{d} \mu^{\prime} .
$$

3. The energy balance equation

$$
\begin{aligned}
& \frac{1}{2 \pi} \sum_{i=1}^{2} \int_{0}^{\infty} \mathrm{d} v \int_{0}^{2 \pi} \mathrm{d} \varphi \\
& \times \int_{-1}^{+1}\left(\left(k_{v}^{i}+\sigma_{v}^{i}\right) I_{\nu}^{i}(\mu, \varphi)-\eta_{v}^{i}(\mu, \varphi)\right) \mathrm{d} \mu=0
\end{aligned}
$$

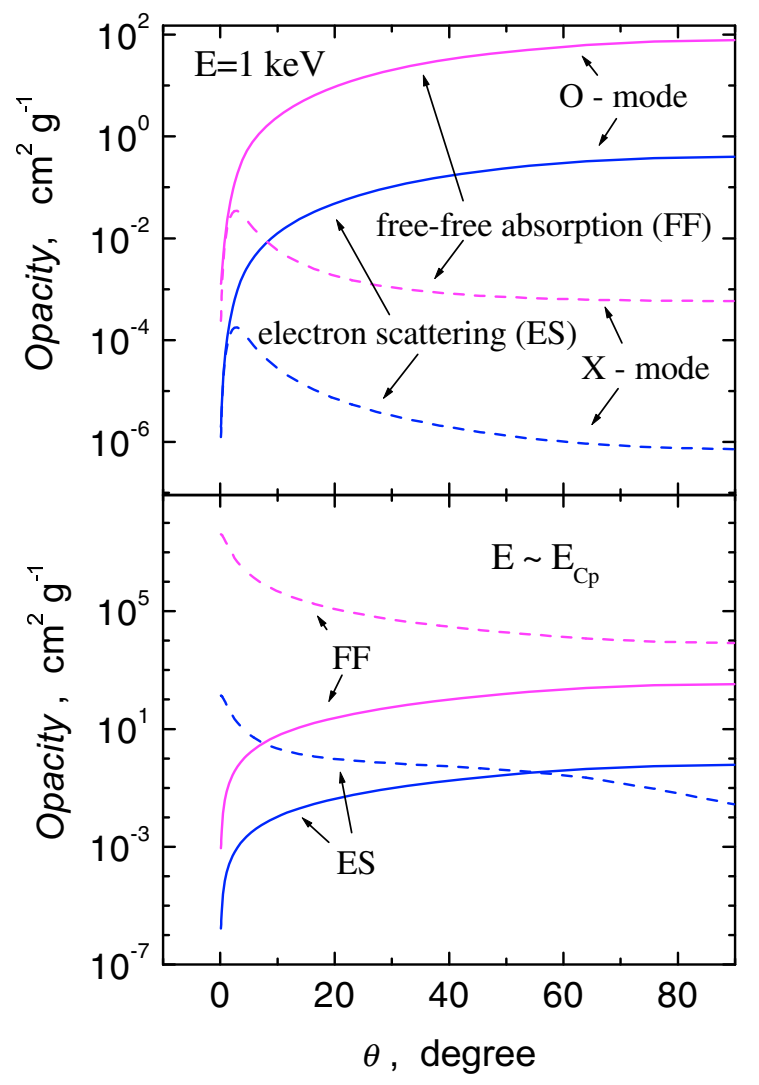

Fig. 3. Dependence of the free-free and electron scattering opacities in two modes on the angle between the magnetic field lines and the direction of photon propagation for two photon energies: $1 \mathrm{keV}$ and at the proton cyclotron energy. Plasma parameters and magnetic field strength are the same as in Fig. 2.

with emissivity

$$
\begin{aligned}
\eta_{\nu}^{i}(\mu, \varphi) & =\frac{1}{2 \pi} \sum_{j=1}^{2} \int_{0}^{2 \pi} \mathrm{d} \varphi^{\prime} \int_{-1}^{+1} \sigma_{v}^{i j}\left(\mu, \varphi ; \mu^{\prime}, \varphi^{\prime}\right) I_{v}^{j}\left(\mu^{\prime}, \varphi^{\prime}\right) \mathrm{d} \mu^{\prime} \\
& +k_{v}^{i} \frac{B_{v}}{2} .
\end{aligned}
$$

Equations (1)-(10) must be completed by the EOS and the charge and particle conservation laws. In the code two different cases of these laws are considered.

In the first (simplest) case, a fully ionized atmosphere are calculated. Therefore, the EOS is the ideal gas law

$P_{\mathrm{g}}=n_{\mathrm{tot}} k T$,

where $n_{\text {tot }}$ is the number density of all particles. Opacities are calculated in the same way as in the paper by van Adelsberg \& Lai (2006) (see references therein for the background theory and more sophisticated approaches). The vacuum polarization effect is taken into account following the same work. Examples of opacities as functions of photon energy and angle $\theta$ for a magnetized electron-proton plasma are shown in Figs. 2 and 3.

The second considered case is a partially ionized hydrogen atmosphere. In this case the EOS and the corresponding opacities are taken from tables calculated by Potekhin \& Chabrier (2003, 2004). The normal mode polarization vectors are taken from the calculations by Potekhin et al. (2004). The vacuum polarization effect is also included.

For solving the above equations and computing the model atmosphere, we used a version of the computer code ATLAS 


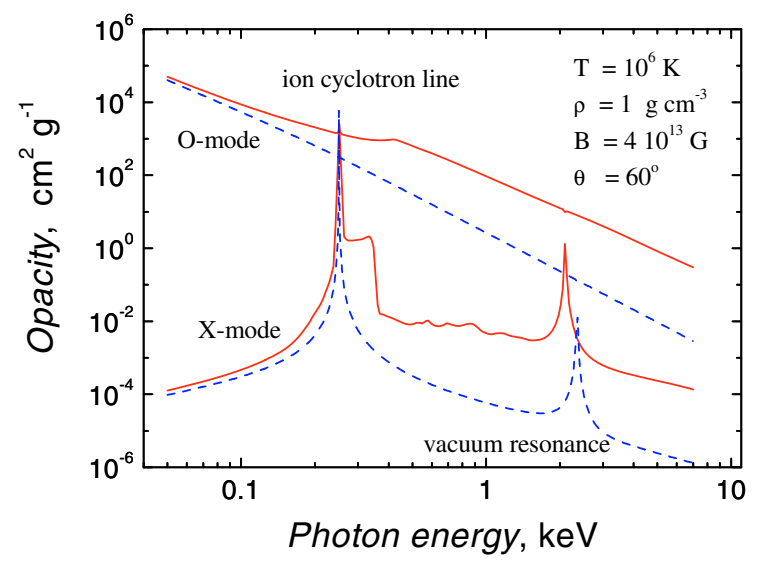

Fig. 4. Dependence of the true opacities in two modes on the photon energy for fully (dashed curves) and partially ionized (solid curves) hydrogen. The vacuum polarization effect is taken into account. The plasma temperature is $10^{6} \mathrm{~K}$, the plasma density is $1 \mathrm{~g} \mathrm{~cm}^{-3}$, the magnetic field strength is $4 \times 10^{13} \mathrm{G}$. The angle between photon propagation direction and the magnetic field is $60^{\circ}$.

(Kurucz 1970, 1993), modified to deal with strong magnetic fields. A nonmagnetic version of this modified code was previously used to model atmospheres of super-soft X-ray sources (Swartz et al. 2002; Ibragimov et al. 2003), atmospheres of non-magnetized NSs (Suleimanov \& Werner 2007; Rauch et al. 2008), and atmospheres of spreading layers on the surface of accreting NSs (Suleimanov \& Poutanen 2006).

The scheme of calculations is as follows. First of all, the input parameters of the model atmosphere are defined: $T_{\text {eff }}, g$, $\boldsymbol{B}$ and the chemical composition. Then a starting model using a grey temperature distribution is calculated. The calculations are performed with a set of 90 depth points $m_{j}$ distributed logarithmically in equal steps from $m_{1} \approx 10^{-5} \mathrm{~g} \mathrm{~cm}^{-2}$ to $m_{\max } \approx$ $10^{5} \mathrm{~g} \mathrm{~cm}^{-2}$ in the case of a semi-infinite atmosphere. It is also possible to calculate thin atmospheres with arbitrary values of $m_{\max }$. In this case the temperature at the inner boundary is considered as the temperature of a condensed NS surface.

In the starting model, all number densities and opacities at all depth points and all photon energies are calculated. We use 200 logarithmically equidistant energy points in our computations in the range $0.001-20 \mathrm{keV}$ with 9 additional points near each ion cyclotron resonance

$E_{\mathrm{ci}}=0.635 \mathrm{keV} \frac{B}{10^{14} \mathrm{G}} \frac{Z}{A}$,

where $Z$ is the ionic charge and $A$ the atomic weight in the atomic units. If the vacuum resonance is taken into consideration, then another photon energy grid is used, which is constructed using the "equal grid" method (Ho \& Lai 2003). In this method every point in the depth grid $m_{j}$ corresponds to the point in the energy grid defined by the equation

$E_{i}=E_{\mathrm{V}}\left(\rho\left(m_{j}\right)\right)$

(if this energy point is in the considered energy range $0.001-20 \mathrm{keV})$. Here $E_{\mathrm{V}}(\rho)$ is the energy of the vacuum resonance at given $B$ and $\rho$ (see van Adelsberg \& Lai 2006). The opacity averaged in the energy interval $\left(E_{i-1}, E_{i+1}\right)$ is used to avoid opacity overestimation in frequency integrals like (9). This energy grid is recalculated after every iteration. Note that the vacuum resonance energy $E_{\mathrm{V}}(\rho)$ in a partially ionized plasma is shifted relative to its value in a fully ionized plasma (see Fig. 4).
The radiation transfer Eq. (5) is solved on a set of 40 polar angles $\theta$ and 6 azimuthal angles $\varphi$ (in the case of inclined magnetic field) by the short characteristic method (Olson \& Kunasz 1987).

We use the conventional condition (no external radiation) at the outer boundary

$I_{v}^{i}\left(\mu<0, m=m_{1}\right)=0$.

The diffusion approximation is used as the inner boundary condition

$I_{v}^{i}\left(\mu>0, m=m_{\max }\right)=\frac{B_{v}}{2}$.

The code allows one to take into account the partial mode conversion according to van Adelsberg \& Lai (2006). At the vacuum resonance, the intensity in the extraordinary mode partially converts with the probability $1-P_{\text {jump }}$ to the intensity in the ordinary one and vice versa

$I_{v}^{1,2} \rightarrow P_{\text {jump }} I_{v}^{1,2}+\left(1-P_{\text {jump }}\right) I_{v}^{2,1}$,

where

$P_{\text {jump }}=\exp \left[-\frac{\pi}{2}\left(E / E_{\mathrm{ad}}\right)^{3}\right]$.

The value $E_{\text {ad }}$ is a function of $E, B, \rho, T, \mu$ (see van Adelsberg \& Lai 2006 for details).

The solution of the radiative transfer Eq. (5) is checked for the energy balance Eq. (9), together with the surface flux condition

$4 \pi \int_{0}^{\infty} H_{v}(m=0) \mathrm{d} v=\sigma T_{\text {eff }}^{4}=4 \pi H_{0}$,

where the Eddington flux at any given depth $m$ is defined as

$H_{v}(m)=\frac{1}{4 \pi} \sum_{i=1}^{2} \int_{0}^{2 \pi} d \varphi \int_{-1}^{+1} \mu I_{v}^{i}(\mu, \varphi, m) \mathrm{d} \mu$.

The relative flux error

$\varepsilon_{H}(m)=1-\frac{H_{0}}{\int_{0}^{\infty} H_{v}(m) d v}$,

and the energy balance error as functions of depth

$$
\begin{aligned}
\varepsilon_{\Lambda}(m)= & \sum_{i=1}^{2} \int_{0}^{\infty} \mathrm{d} v \int_{0}^{2 \pi} \mathrm{d} \varphi \\
& \times \int_{-1}^{+1}\left(\left(k_{v}^{i}+\sigma_{v}^{i}\right) I_{v}^{i}(\mu, \varphi)-\eta_{v}^{i}(\mu, \varphi)\right) \mathrm{d} \mu
\end{aligned}
$$

are calculated.

Temperature corrections are then evaluated using three different procedures. The first is the integral $\Lambda$-iteration method, modified for the two-mode radiation transfer, based on the energy balance Eq. (9). In this method the temperature correction for a particular depth is found from

$\Delta T_{\Lambda}=\frac{-\varepsilon_{\Lambda}(m)}{\int_{0}^{\infty} \Phi_{v} \mathrm{~d} v}$

where

$\Phi_{v}=\sum_{i=1}^{2}\left[\left(\Lambda_{v \text { diag }}^{i}-1\right) /\left(1-\alpha_{v}^{i} \Lambda_{v \text { diag }}^{i}\right)\right] \bar{k}_{v}^{i}\left(\mathrm{~d} B_{v} / \mathrm{d} T\right)$ 

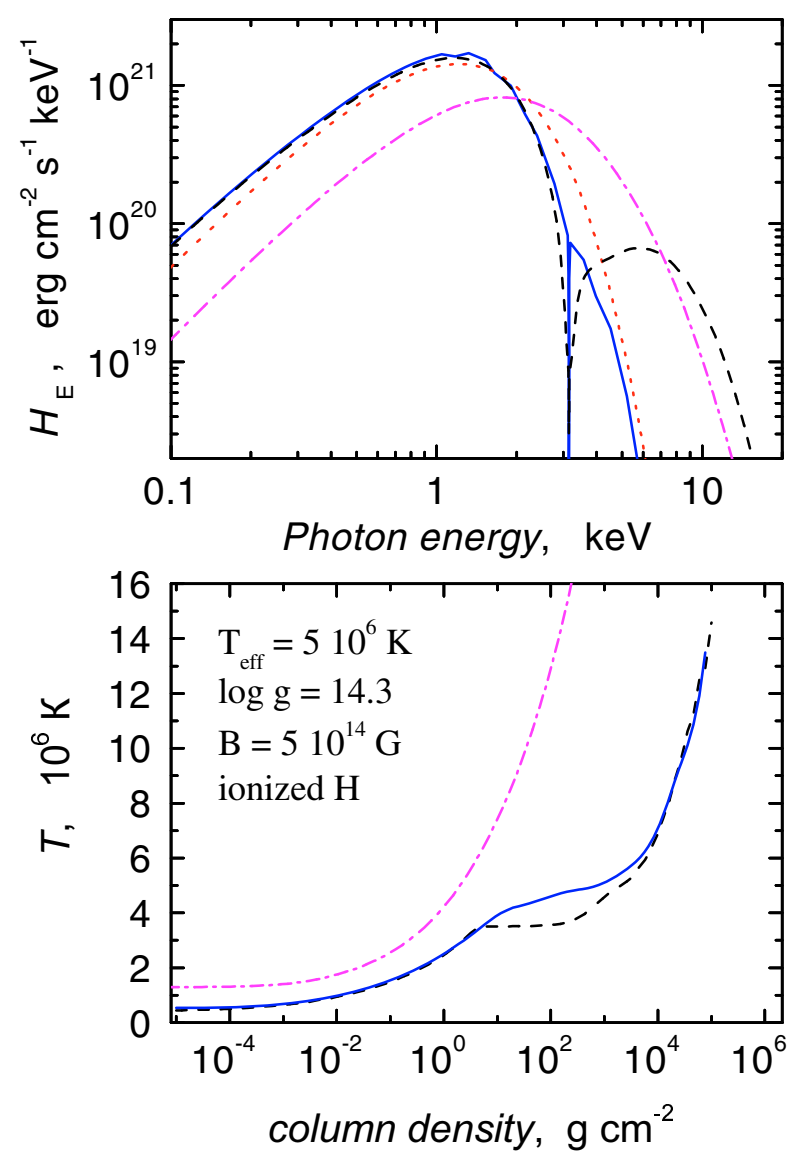

Fig. 5. Emergent spectra and temperature structures of fully ionized hydrogen model atmospheres of neutron stars with $T_{\text {eff }}=5 \times 10^{6} \mathrm{~K}$ and $\log g=14.3$. The models with and without (dash-dotted curves) strong $\left(B=5 \times 10^{14} \mathrm{G}\right)$ magnetic field as well as with (solid curves) and without (dashed curves) vacuum polarization effect are shown. The blackbody spectrum with the temperature equal the effective temperature is also shown in the upper panel (dotted curve).

and $\alpha_{v}^{i}=\bar{\sigma}_{v}^{i} /\left(\bar{k}_{v}^{i}+\bar{\sigma}_{\mu}^{i}\right)$. Averaged opacities are defined as

$$
\begin{aligned}
\bar{k}_{v}^{i} & =\frac{1}{4 \pi} \int_{0}^{2 \pi} \mathrm{d} \varphi \int_{-1}^{+1} k_{v}^{i}(\mu, \varphi) \mathrm{d} \mu, \\
\bar{\sigma}_{v}^{i} & =\frac{1}{4 \pi} \int_{0}^{2 \pi} \mathrm{d} \varphi \int_{-1}^{+1} \sigma_{v}^{i}(\mu, \varphi) \mathrm{d} \mu .
\end{aligned}
$$

$\Lambda_{\nu \text { diag }}^{i}$ is the diagonal matrix element of the $\Lambda$ operator depending on the mean optical depth in given mode $i$. The mean optical depth is defined by $\mathrm{d} \bar{\tau}_{v}^{i}=\left(\bar{k}_{v}^{i}+\bar{\sigma}_{\mu}^{i}\right) \mathrm{d} m$ (see Kurucz 1970 for details of $\Lambda_{v \text { diag }}-\tau_{v}$ dependence). This procedure is used in the upper atmospheric layers. The second procedure is the AvrettKrook flux correction, which uses the relative flux error $\varepsilon_{H}(m)$, and it is performed in the deep layers. And the third one is the surface correction, which is based on the emergent flux error (see Kurucz 1970 for a detailed description).

The iteration procedure is repeated until the relative flux error is smaller than $1 \%$ and the relative flux derivative error is smaller than $0.01 \%$. As a result of these calculations, we obtain a self-consistent isolated NS model atmosphere, together with the emergent spectrum of radiation.

Our method of calculation has been tested by a comparison to models for magnetized NS atmospheres (Shibanov et al. 1992; Pavlov et al. 1994; Ho \& Lai 2001; Özel 2001; Ho \& Lai 2003).
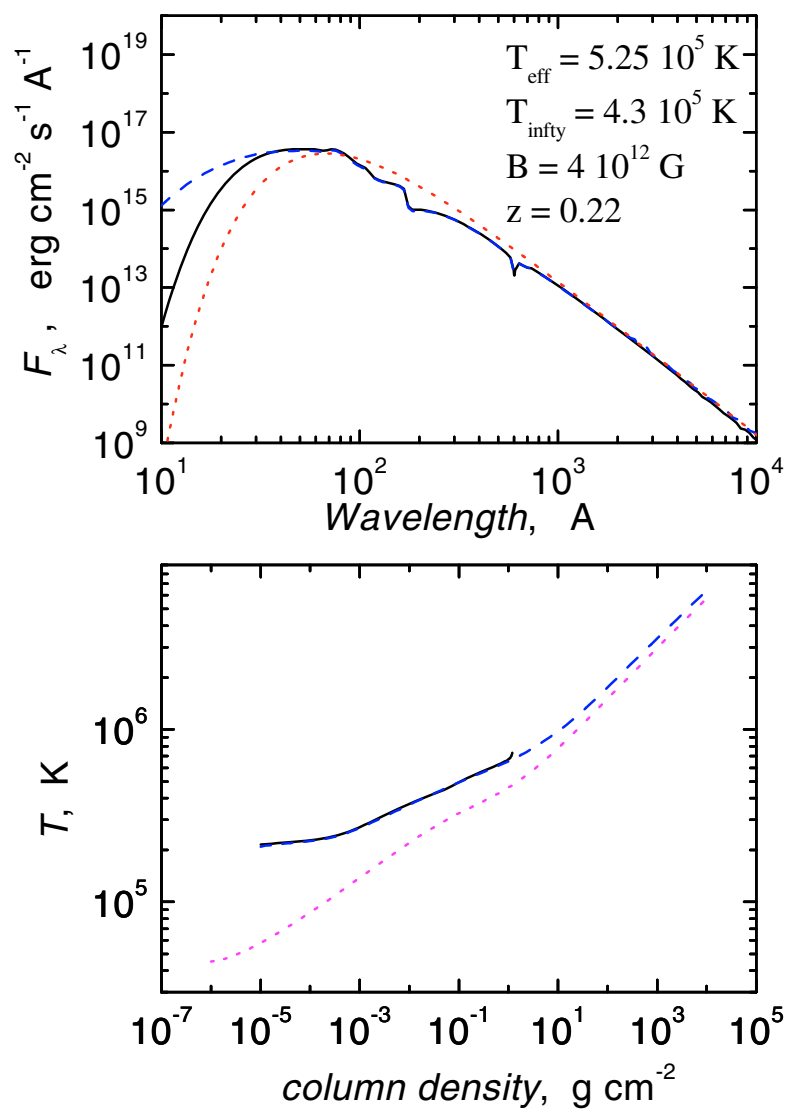

Fig. 6. Top panel: emergent spectra of the partially ionized hydrogen semi-infinite (dashed curve) and thin $\left(\Sigma=1.2 \mathrm{~g} \mathrm{~cm}^{-2}\right.$, solid curve) model atmospheres $\left(T_{\text {eff }}=5.25 \times 10^{6} \mathrm{~K}, B=4 \times 10^{12} \mathrm{G}\right)$ together with the corresponding blackbody spectrum (dotted curve). The spectra are calculated with gravitational redshift $(z=0.22)$ taking into consideration. Bottom panel: temperature structures of the models from the top panel together with the temperature structure of a fully ionized hydrogen model (dotted curve).

Model atmospheres with partially ionized hydrogen are compared to models computed by Ho et al. (2007). We have found that our models are in a good agreement with these calculations. Our results are presented in Fig. 5, where the temperature structures and the emergent spectra of models with and without vacuum polarization are compared to a model without magnetic field. In Fig. 6 we present emergent spectra and temperature structures of the semi-infinite and thin model atmospheres with the same parameters as used by Ho et al. (2007).

\section{Results}

In this work we use the developed code mainly for studies of thin atmospheres above a condensed NS surface. In all calculations below we use the same surface gravity, $\log g=14.3$.

One of the problem related to magnetars (in particular, AXPs) is the lack of any absorption feature at the proton cyclotron energy, although early models of the magnetized NS atmospheres predicted a strong feature at the magnetar field strengths (Ho \& Lai 2001; Zane et al. 2001). Ho \& Lai (2003) suggested that a possible solution of this problem is the suppression of the cyclotron absorption feature due to the vacuum polarization. We confirm this result (see Fig. 5). In addition, we demonstrate that this absorption line is further reduced in a thin atmosphere without vacuum polarization. Figure 7 demonstrates 


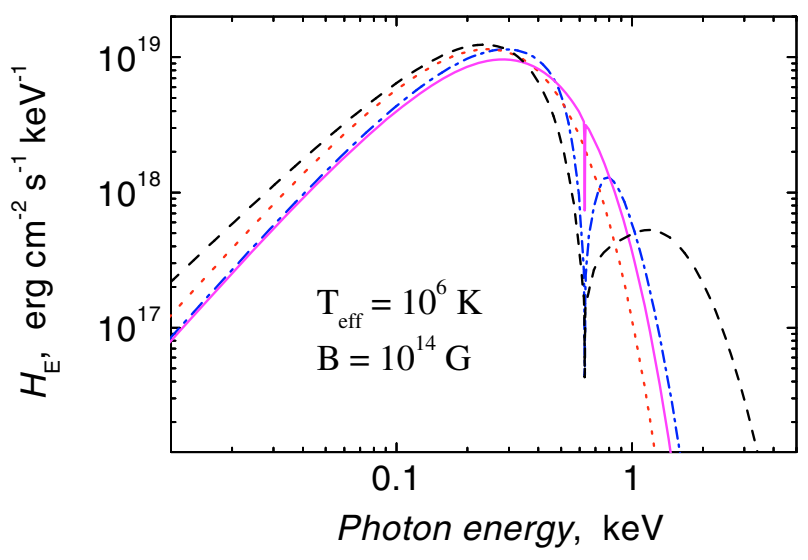

Fig. 7. Emergent spectra of the thin fully ionized hydrogen atmospheres above a solid surface with $T_{\text {eff }}=10^{6} \mathrm{~K}$ and $B=10^{14} \mathrm{G}$ in comparison to the semi-infinite atmosphere (dashed curve). The spectra of atmospheres with surface densities $\Sigma=1$ (solid curve) and $100 \mathrm{~g} \mathrm{~cm}^{-2}$ (dash-dotted curve) together with the corresponding blackbody (dotted curve) are shown. Vacuum polarization effect is not included.

emergent spectra of thin atmospheres without allowance for the vacuum polarization effect. In the semi-infinite atmosphere, a wide proton cyclotron line forms in agreement with the results of Ho \& Lai (2001); Zane et al. (2001). However, the absorption feature disappears with decreasing the atmosphere surface density $\Sigma$. The thin atmosphere is transparent to the continuum and absorption line wings, therefore the emergent spectrum approaches the spectrum of the condensed surface everywhere except for a narrow energy band at the center of the cyclotron line. The width of this absorption depends on the atmosphere thickness.

Some XDINSs and CCOs show one or two absorption features (Haberl 2007; Sanwal et al. 2002; Schwope et al. 2007). Various hypothesis (so far inconclusive) were considered for an explanation (see Mori \& Ho 2007 and references therein). Here we suggest another one, which we name "sandwich atmosphere". A thin, chemically layered atmosphere above a condensed NS surface can arise from accretion of interstellar gas with cosmic chemical composition. In this case, hydrogen and helium quickly separate due to the high gravity (according to Brown et al. 2002, the $\mathrm{He} / \mathrm{H}$ stratification timescale can be estimated as $\sim \rho_{1}^{1.3} T_{6}^{-0.3} g_{14}^{-2} \mathrm{~s}$, where $\rho_{1}=\rho / 10 \mathrm{~g} \mathrm{~cm}^{-3}, T_{6}=$ $T / 10^{6} \mathrm{~K}$, and $g_{14}=g / 10^{14} \mathrm{~cm} \mathrm{~s}^{-2}$ ). In this "sandwich atmosphere" a layer of hydrogen is located above a helium slab, and the emergent spectrum has two absorption features, corresponding to proton and $\alpha$-particle cyclotron energies. In Fig. 8 the emergent spectrum for one of these models is shown. The emission feature at the helium absorption line arises due to a local temperature bump at the boundary between the helium and hydrogen layers. This bump is due to sharp changes of the plasma density and the opacity between helium and hydrogen layers. Clearly, some transition zone with mixed H/He chemical composition must exist between layers, and this rapid temperature change should be reduced. We plan to calculate models with this kind of transition zone in future work.

For comparison to observations, it is necessary to integrate the local model spectra over the NS surface. The effective temperature and magnetic field strengths are not uniform over the NS surface, and generally the magnetic field is not perpendicular to the surface (see Ho et al. 2008). Therefore, it is necessary to compute model atmospheres with inclined magnetic field. This possibility is included in our code. For example, Fig. 9 shows
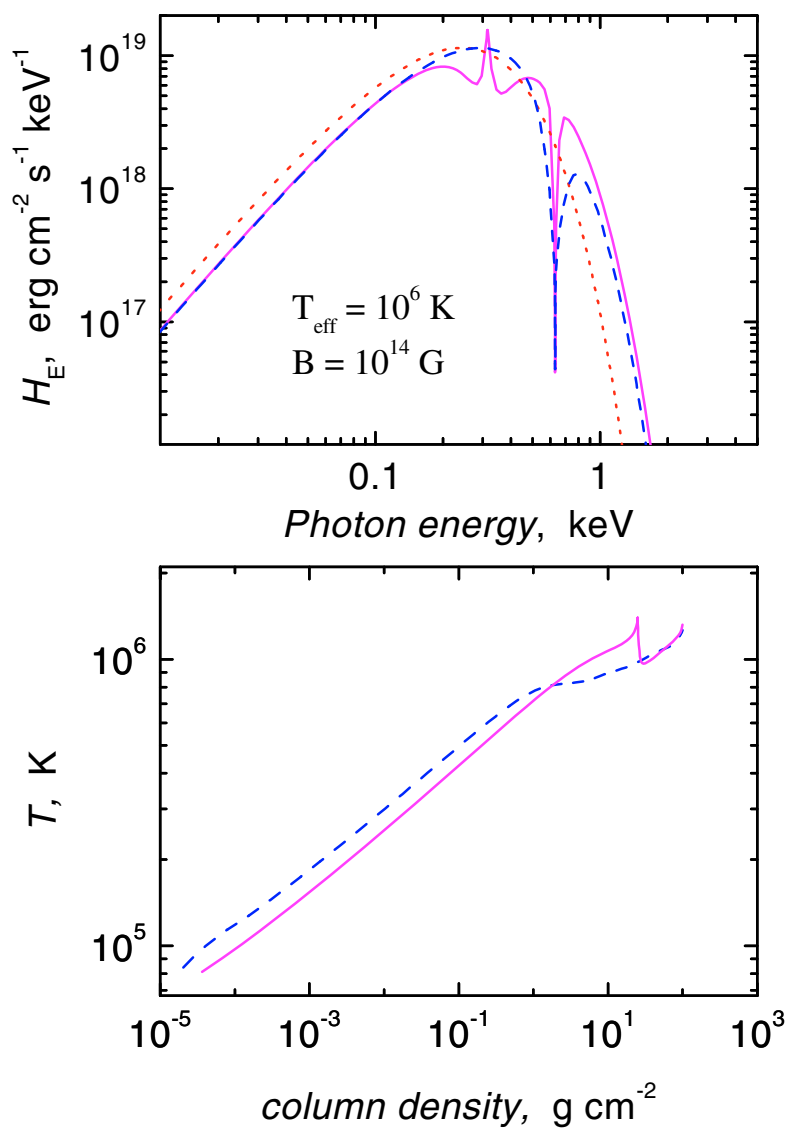

Fig. 8. Emergent spectrum (top panel) and temperature structure (bottom panel) of the "sandwich" model atmosphere above a solid surface with $T_{\text {eff }}=10^{6} \mathrm{~K}$ and $B=10^{14} \mathrm{G}$ (solid curve) in comparison with the thin fully ionized hydrogen atmosphere (dashed curve) with the same parameters. The surface densities of both model atmospheres are $100 \mathrm{~g} \mathrm{~cm}^{-2}$, in the "sandwich" model the $\mathrm{H}$ slab has $25 \mathrm{~g} \mathrm{~cm}^{-2}$ surface density and the He slab has $75 \mathrm{~g} \mathrm{~cm}^{-2}$. The corresponding blackbody spectrum (dotted curve) is also shown in the top panel.

spectra and temperature structures of model atmospheres with magnetic field perpendicular and parallel to the NS surface.

Most of the XDINSs have magnetic fields $B \geq 10^{13} \mathrm{G}$ and color temperatures $\approx 10^{6} \mathrm{~K}$ (Haberl 2007). Hydrogen model atmospheres are partially ionized under these conditions and the vacuum polarization effect is also significant. Here we present first results of modeling of partially ionized hydrogen atmospheres using our radiative transfer code. In Fig. 10 we compare spectra and temperature structures of the partially ionized hydrogen model atmospheres with and without the partial mode conversion effect. When the X-mode (having smaller opacity) partially converts to the O-mode in the surface layers of the atmosphere, the energy absorbed by the O-mode heats these upper layers. As a result, the emergent spectra are closer to the blackbody.

For some of the XDINSs, optical counterparts have been found (Mignani et al. 2007). The observed optical fluxes are a few times larger compared to the blackbody extrapolation from $\mathrm{X}$-rays to the optical range (see top panel of Fig. 12 for illustration). Ho et al. (2007) demonstrated, that a single partially ionized thin hydrogen atmosphere can explain this problem in the case of brightest isolated NS RX J1856.4-3754: the model fits well both, the observed optical flux and the X-ray spectrum. RXJ1856.4-3754 has very low pulsed fraction of 

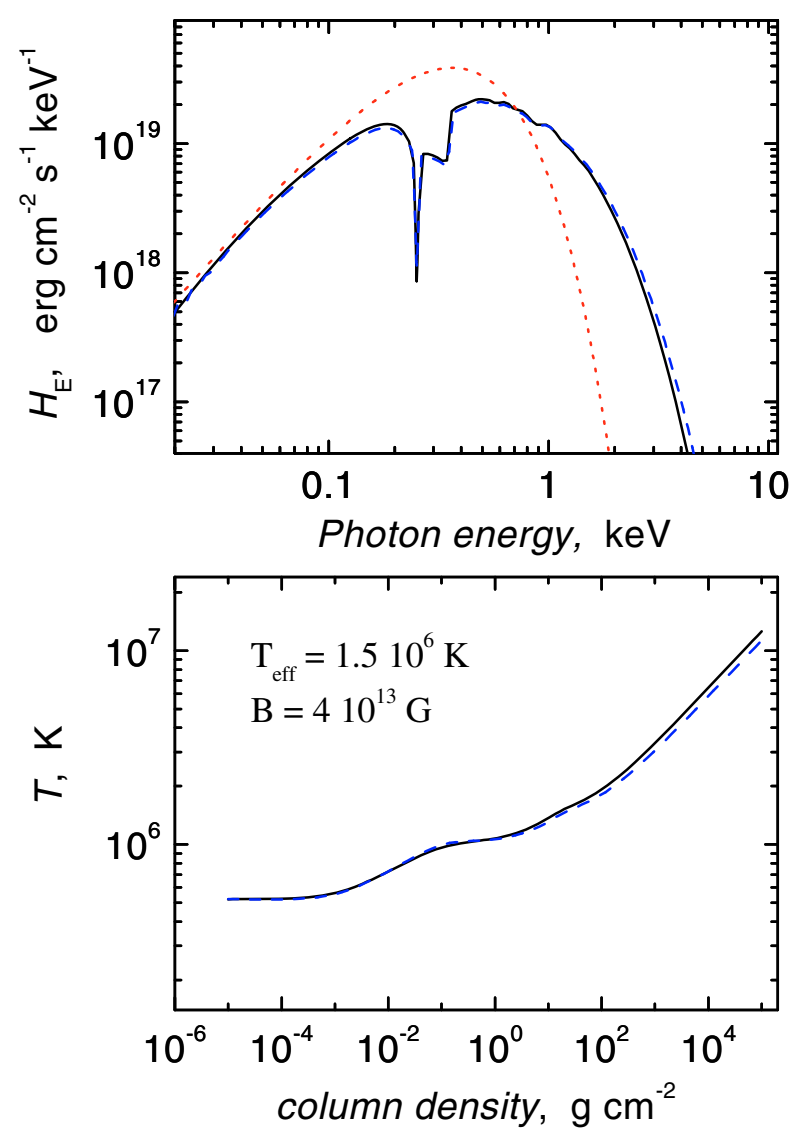

Fig. 9. Emergent spectra and temperature structures of partially ionized hydrogen model atmospheres with $T_{\text {eff }}=1.5 \times 10^{6} \mathrm{~K}$ with inclinations of the magnetic field $\left(B=4 \times 10^{13} \mathrm{G}\right)$ to the surface normal $\theta_{B}$ equal $0^{\circ}$ (solid curves) and $90^{\circ}$ (dashed curves). The vacuum polarization effect is not included. The corresponding blackbody spectrum is also shown in the upper panel (dotted curve).

radiation $(\approx 1.2 \%$, Tiengo \& Mereghetti 2007), therefore it is possible to fit the radiation of this star by the single model atmosphere. Other XDINSs have larger pulse fractions, up to $18 \%$ (RBS 1223, Haberl et al. 2004). In this case the temperature distribution across the NS surface is not homogenous, and the excess optical flux can be explained by the radiation from cool surface parts (Schwope et al. 2005).

We now investigate properties of partially ionized hydrogen modes, which can be applied to the RBS 1223 atmosphere. The color temperature of this star, found from $\mathrm{X}$-ray spectra fitting, is close to $10^{6} \mathrm{~K}$, with magnetic field $B \approx 4 \times 10^{13} \mathrm{G}$ (Schwope et al. 2007). In particular, we investigate the optical flux excess in comparison to the X-ray fitted blackbody flux in this kind of models. For this aim we have calculated two sets of models with vacuum polarization and partial mode conversion. The models in the first set have effective temperatures $T_{\text {eff }}=10^{6} \mathrm{~K}$ and the models of second one have effective temperatures $T_{\text {eff }}=1.2 \times 10^{6} \mathrm{~K}$. In both sets $B=4 \times 10^{13} \mathrm{G}$, and models with surface densities $\Sigma=1,3,10,30,100$ and $10^{5}$ (semi-infinite model) $\mathrm{g} \mathrm{cm}^{-2}$ are computed. In Fig. 11 we show emergent spectra and temperature structures for some models from the second set. Clearly, the X-ray spectra of the models with $\Sigma \leq 10 \mathrm{~g} \mathrm{~cm}^{-2}$ are close to a blackbody and, therefore, better fit the observed $\mathrm{X}$-ray spectrum.

In Fig. 12 (bottom panel) we show the ratio of the model atmosphere flux to the X-ray fitted blackbody flux in the optical
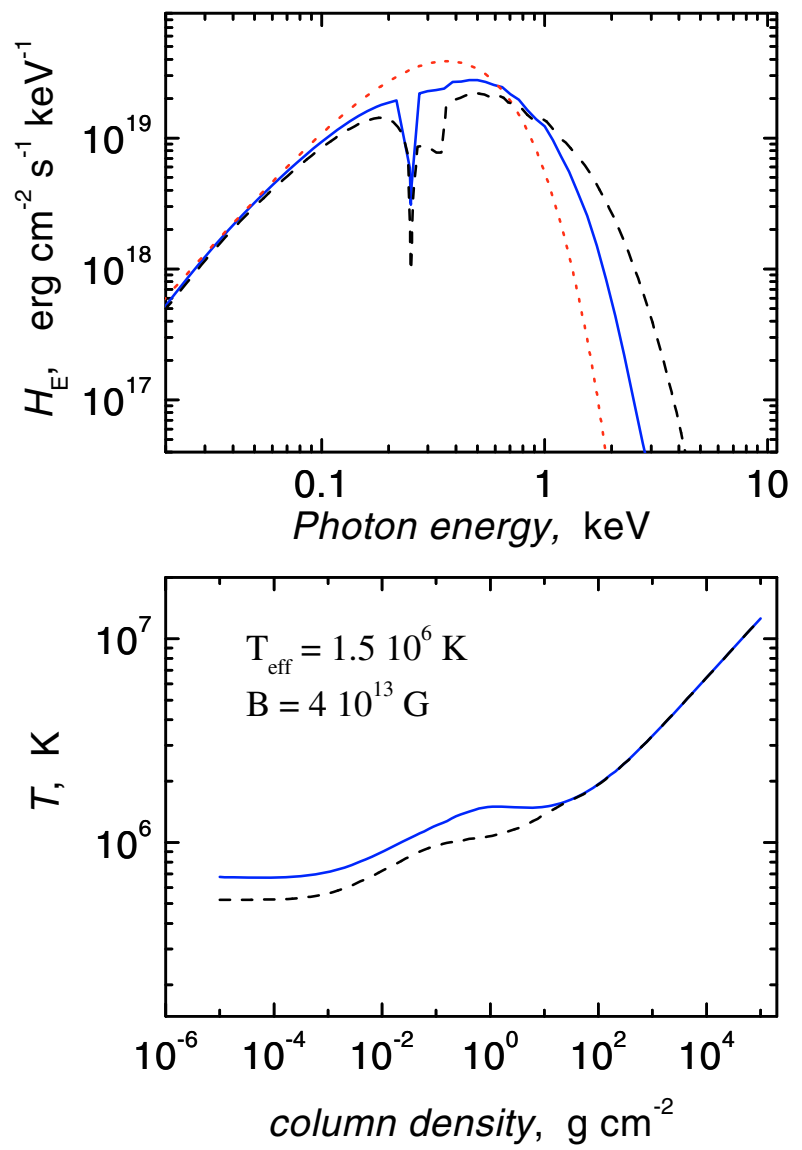

Fig. 10. Emergent spectra and temperature structures of the partially ionized hydrogen model atmospheres with $T_{\text {eff }}=1.5 \times 10^{6} \mathrm{~K}$ with (solid curves) and without (dashed curves) vacuum polarization effect (with the partial mode conversion). The magnetic field strength is $B=4 \times$ $10^{13} \mathrm{G}$. The corresponding blackbody spectrum is also shown in the upper panel (dotted curve).

band depending on $\Sigma$ for both sets. The observed ratio is about 5 (Kaplan et al. 2002b), in agreement with the semi-infinite atmosphere models. However, the observed blackbody like X-ray spectrum agrees with the thin atmosphere models, for which this ratio is close to 1 . Therefore, the observed optical excess cannot be explained by the thin atmosphere model alone; instead, it can arise due to a nonuniform surface temperature distribution, in agreement with the RBS 1223 light curve modeling (Schwope et al. 2005).

\section{Conclusions}

In this paper a new code for the computation of magnetized NS model atmosphere is presented. It can model fully ionized and partially ionized hydrogen atmospheres in a wide range of effective temperatures $\left(3 \times 10^{5}-10^{7} \mathrm{~K}\right)$ and magnetic fields $\left(10^{12}\right.$ $\left.10^{15} \mathrm{G}\right)$, with any inclination of the magnetic field to the stellar surface. The vacuum polarization effect with partial mode conversion is taken into consideration. Calculated emergent spectra and temperature structures of the model atmospheres agree with previously published ones.

We presented new results obtained using this code. We have studied the properties of thin atmospheres above condensed NS surfaces. We demonstrated that the proton cyclotron absorption line disappears in the thin hydrogen model atmospheres. A new thin "sandwich" model atmosphere (hydrogen layer above 

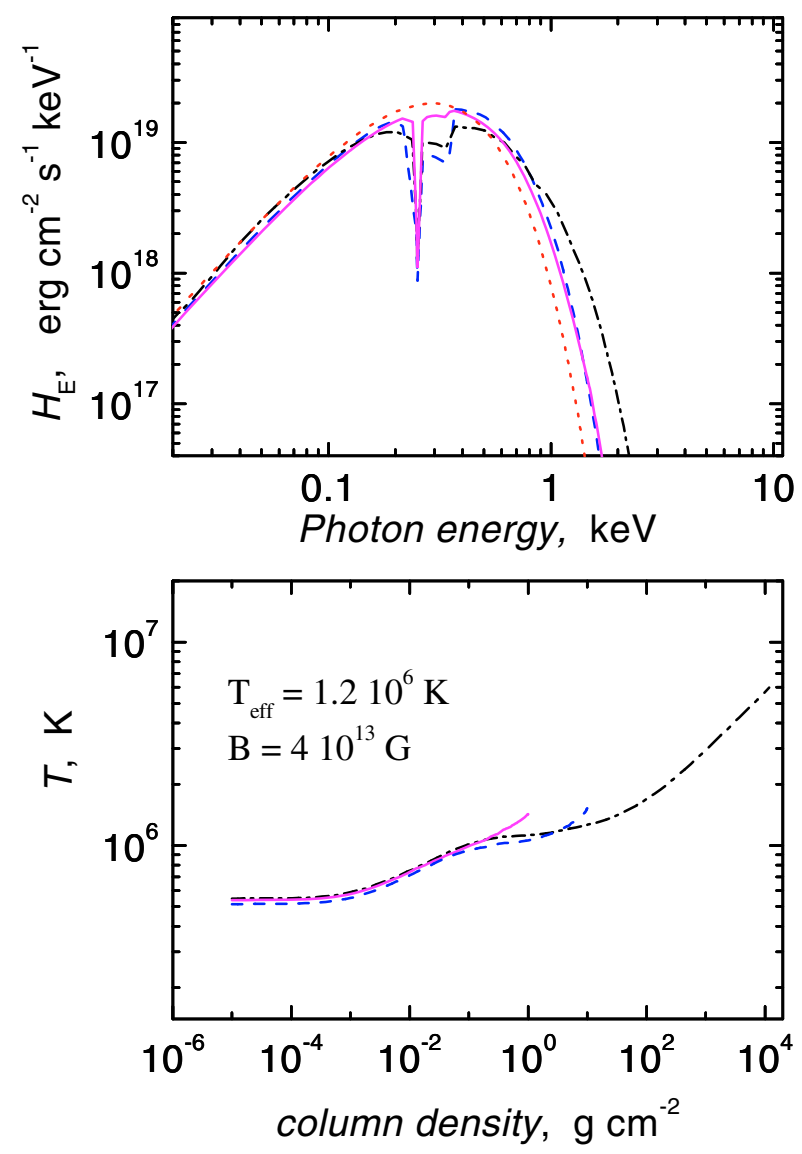

Fig. 11. Emergent spectra and temperature structures of the partially ionized hydrogen model atmospheres with $T_{\text {eff }}=1.2 \times 10^{6} \mathrm{~K}$ with vacuum polarization effect the partial mode conversion shown for various surface densities $\Sigma$ (solid curves $-1 \mathrm{~g} \mathrm{~cm}^{-2}$, dashed curves $-10 \mathrm{~g} \mathrm{~cm}^{-2}$, dash-dotted curves - semi-infinite atmosphere). The magnetic field strength is $B=4 \times 10^{13} \mathrm{G}$. The corresponding blackbody spectrum is also shown in the upper panel (dotted curve).

helium layer) is proposed to explain the occurrence of two absorption features in the observed X-ray spectra of some isolated NSs.

We analyzed the optical excess (relative to the X-ray fitted blackbody flux) in the model spectra of partially ionized hydrogen atmospheres with vacuum polarization and partial mode conversion.

A set of model atmospheres with parameters (effective temperature and the magnetic field strength) close to the probable parameters of the isolated NS RBS 1223 were calculated. We found the optical flux excess $\approx 5$ for the semi-infinite model atmospheres decreases down to 1 with decreasing surface density $\Sigma$ of the atmosphere. Spectra of thin model atmospheres fit the observed RBS 1223 X-ray spectrum better, therefore we conclude that the observed optical excess should be explained by nonuniform surface temperature distribution.

The accuracy of the thin and sandwich model atmospheres is currently limited by the inner boundary condition for the radiation transfer equation. We used blackbody radiation as this condition, but a higher accuracy can be achieved by replacing it by the condensed surface condition (van Adelsberg et al. 2005). This will be done in a future work. We are also planning to include the effect of magnetic field and temperature distributions over the stellar surface to compute an integral emergent spectrum from isolated NSs.
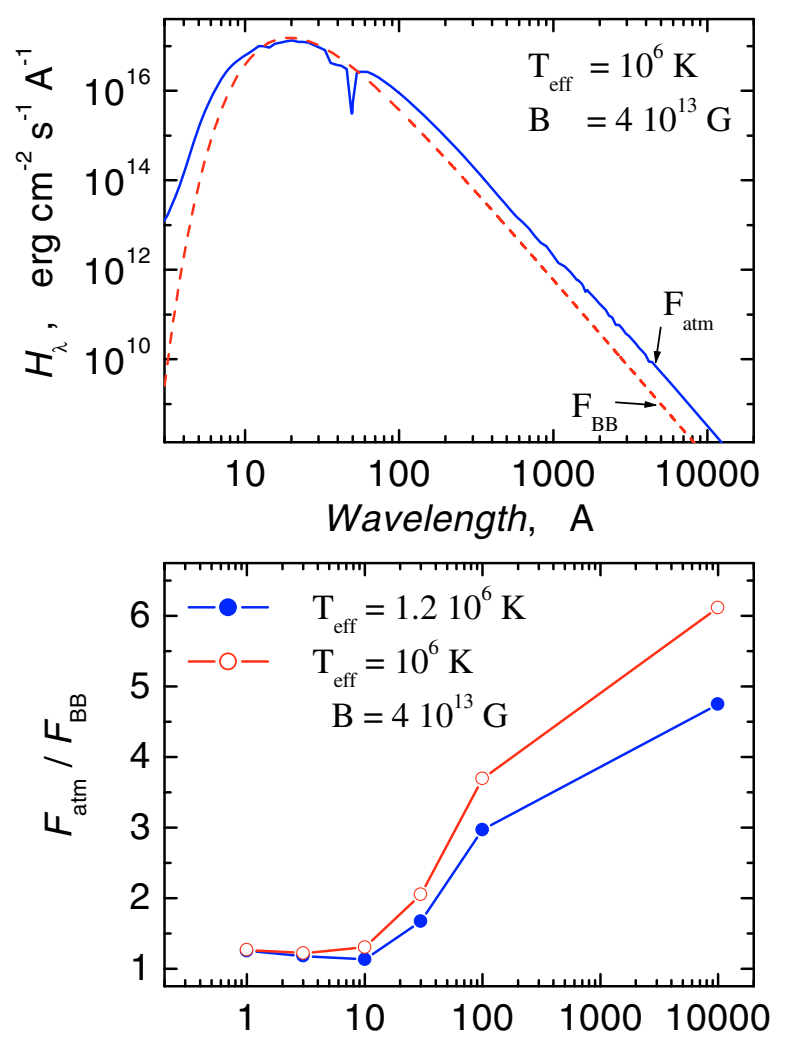

Atmosphere surface density $\Sigma, \mathrm{g} \mathrm{cm}^{-2}$

Fig. 12. Top panel: emergent spectrum of the partially ionized hydrogen model atmosphere of neutron stars with $T_{\text {eff }}=10^{6} \mathrm{~K}$ with vacuum polarization effect, partial mode conversion and magnetic field strength $B=4 \times 10^{13} \mathrm{G}$. The blackbody spectrum fitted to the maximum flux of the spectral distribution is also shown (dashed curve). At the optical band the model atmosphere flux in a few times larger than the blackbody flux. Bottom panel: ratios of the model atmosphere flux to the blackbody (X-ray fitted) flux at the optical band depending on the model atmosphere thickness (surface density $\Sigma$ ) for the models with $T_{\text {eff }}=10^{6} \mathrm{~K}$ and $T_{\text {eff }}=1.2 \times 10^{6} \mathrm{~K}$.

Acknowledgements. VS thanks DFG for financial support (grant We 1312/351 and grant SFB/Transregio 7 "Gravitational Wave Astronomy") and the President's programme for support of leading science schools (grant NSh4224.2008.2). The work of A.Y.P. is supported by RFBR grants 05-02-2203 and 08-02-00837 and the President's programme for support of leading science schools (grant NSh-2600.2008.2).

\section{References}

Baiotti, L., Giacomazzo, B., \& Rezzolla, L. 2008, Phys. Rev. D, 78, 084033

Brown, E. F., Bildsten, L., \& Chang, P. 2002, ApJ, 574, 920

Burwitz, V., Zavlin, V. E., Neuhäuser, R., et al. 2001, A\&A, 379, L35

Burwitz, V., Haberl, F., Neuhäuser, R., et al. 2003, A\&A, 399, 1109

Gänsicke, B. T., Braje, T. M., \& Romani, R. W. 2002, A\&A, 386, 1001

Ginzburg, V. L. 1970, The Propagation of Electromagnetic Waves in Plasmas, (2nd edn. Oxford: Pergamon)

Haberl, F., Motch, C., Zavlin, V. E. et al. 2004, A\&A, 424, 635

Haberl, F. 2007, A\&SS, 308, 181

Ho, W. C. G., \& Lai, D. 2001, MNRAS, 327, 1081

Ho, W. C. G., \& Lai, D. 2003, MNRAS, 338, 233

Ho, W. C. G., \& Lai, D. 2004, ApJ, 607, 420

Ho, W. C. G., Kaplan, D. L., Chang, P., van Adelsberg, M., \& Potekhin, A. Y. 2007, MNRAS, 375, 821

Ho, W. C. G., Potekhin, A. Y., \& Chabrier, G. 2008, ApJS, 178, 102

Ibragimov, A. A., Suleimanov, V. F., Vikhlinin, A., \& Sakhibullin, N. A. 2003, Astron. Rep., 47, 186

Kaminker, A. D., Pavlov, G. G., \& Shibanov, Yu. A. 1982, Ap\&SS, 86, 249 Kaminker, A. D., Pavlov, G. G., \& Shibanov, Yu. A. 1983, Ap\&SS, 91, 167 
Kaplan, D. L., van Kerkwijk, M. H., Anderson, J. 2002a, ApJ, 571, 447

Kaplan, D. L., Kulkarni, S. R., \& van Kerkwijk, M. H. 2002b, ApJ, 579, L29

Kaplan, D. L., van Kerkwijk, M. H., Marshall, H. L., et al. 2003, ApJ, 590, 1008

Kaspi, V. M. 2007, A\&SS, 308,

Kurucz, R. L. 1970, SAO Spec. Rep., 309

Kurucz, R. 1993, Atomic data for opacity calculations. Kurucz CD-ROMs, Cambridge, Mass.: Smithsonian Astrophysical Observatory, 1

Lai, D. 2001, Rev. Mod. Phys., 73, 629

Lai, D., \& Salpeter, E. E. 1997, ApJ, 491, 270

Lai, D., \& Ho. W. C. G. 2002, ApJ, 566, 373

Lai, D., \& Ho. W. C. G. 2003, ApJ, 588, 962

Lattimer, J. M., \& Prakash, M. 2007, Phys. Rep., 442, 109

Medin, Z., \& Lai, D. 2007, MNRAS, 382, 1833

Mereghetti, S. 2008, A\&A Rev., 15, 225

Mereghetti, S., Tiengo, A., \& Israel, G. L. 2002, ApJ, 569, 275

Mereghetti, S., Esposito, P., \& Tiengo, A. 2007, A\&SS, 308, 13

Mésźaros, P. 1992, High-Energy Radiation from Magnetized Neutron Stars (Chicago: Univ. Chicago Press)

Mignani, R. P., Bagnulo, S., De Luca, A., et al. 2007, A\&SS, 308, 203

Mihalas, D. 1978, Stellar atmospheres, 2nd edition (San Francisco: W. H. Freeman and Co.)

Mori, K., \& Ho, W. C. G. 2007, MNRAS, 377, 905

Motch, C., Zavlin, V. E., \& Haberl, F. 2003, A\&A, 408, 323

Olson, G. L., \& Kunasz, P. B. 1987, JQSRT, 38, 325

Özel, F. 2001, ApJ, 563, 276

Pavlov, G. G., \& Gnedin, Yu. N. 1984, Astrophys. Space Phys. Rev., 3, 197

Pavlov, G. G., \& Mészáros, P. 1993, ApJ, 416, 752

Pavlov, G. G., Shibanov, Yu. A., Ventura, J. \& Zavlin, V. E. 1994, A\&A, 289, 837

Pavlov, G. G., Sanwal, D., \& Garmire, G. P. 2002, In Neutron Stars in Supernova Remnants, ed. P. O. Slane, \& B. M. Gaensler, ASP Conf. Ser. 271, 247

Pavlov, G. G., Sanwal, D., Teter, M. A. 2004, in Young Neutron Stars and Their Enviroments ed. F. Camilo, \& B. M. Gaensler (San Francisco: ASP), Proc. IAU Symp., 218, 239
Pérez-Azorín, J. F., Miralles, J. A., \& Pons, J. A. 2005, A\&A, 433, 275

Pons, J. A., Walter, F. M., Lattimer, J., et al. 2002, ApJ, 564, 981

Potekhin, A. Y., \& Chabrier, G. 2003, ApJ, 585, 955

Potekhin, A. Y., \& Chabrier, G. 2004, ApJ, 600, 317

Potekhin, A. Y., Chabrier, G., \& Shibanov, Yu. A. 1999, Phys. Rev. E, 60, 2193

Potekhin, A. Y., Lai, D., Chabrier G., \& Ho, W. C. G. 2004, ApJ, 612, 1034

Rajagopal, M., \& Romani, R. W. 1996, ApJ, 461, 327

Rajagopal, M., Romani, R. W., \& Miller, M. C. 1997, ApJ, 479, 347

Rauch, T., Suleimanov, V., \& Werner, K. 2008, A\&A, 490, 1127

Romani, R. W. 1987, ApJ, 313, 718

Sanwal, D., Pavlov, G. G., Zavlin, V. E., et al. 2002, ApJ, 574, L61

Schwope, A. D., Hambaryan, V., Haberl, F., et al. 2005, A\&A, 441, 597

Schwope, A. D., Hambaryan, V., Haberl, F., \& Motch, C. 2007, Ap\&SS, 308, 619

Shibanov, I. A., Zavlin, V. E., Pavlov, G. G., \& Ventura, J. 1992, A\&A, 266, 313

Suleimanov, V., \& Poutanen, J. 2006, MNRAS, 369, 2036

Suleimanov, V., \& Werner, K. 2007, A\&A, 466, 661

Swartz, D. A., Ghosh, K. K., Suleimanov, V., Tennant, A. F., \& Wu, K. 2002, ApJ, 574, 382

Tiengo, A., \& Mereghetti, S. 2007, ApJ, 657, L101

Trümper, J. E., Burwitz, V., Haberl, F., \& Zavlin, V. E. 2004, Nucl. Phys. B Proc. Suppl., 132, 560

Turolla, R., Zane, S., \& Drake, J. J. 2004, ApJ, 603, 265

van Adelsberg, M., \& Lai, D. 2006, MNRAS, 373, 1495

van Adelsberg, M., Lai, D., Potekhin, A. Y., \& Arras, P. 2005, ApJ, 628, 902

van Kerkwijk, M. H., \& Kaplan, D. L. 2007, Ap\&SS, 308, 191

Ventura, J. 1979, Phys. Rev. D, 19, 1684

Werner, K., \& Deetjen, J. 2000, in Pulsar Astronomy-2000 and Beyond, ed. M. Kramer, N. Wex, \& R. Wielebinski, ASP Conf. Ser., 202, 623

Zane, S., Turolla, R., Stella, L., \& Treves, A. 2001, ApJ, 560, 384

Zavlin, V. E. 2009, in Neutron Stars and Pulsars, ed. W. Becker (Berlin: Springer), ASSL, 357, 181

Zavlin, V. E., Pavlov, G. G., \& Shibanov, I. A. 1996, A\&A, 315, 141 